\title{
Improvement in energy conversion for unmanned aerial vehicle charging pad
}

\author{
M. R.AL-Obaidi ${ }^{1}$, M. A. Mustafa ${ }^{2}$, W.Z.W. Hassan ${ }^{3}$, N. Azis ${ }^{4}$, A. H. Sabry ${ }^{5}$, \\ Mohd Zainal Abidin Ab-Kadir ${ }^{6}$ \\ ${ }^{1,2,3,4}$ Department of Electrical and Electronic Engineering, Faculty of Engineering, Universiti Putra Malaysia, Malaysia \\ ${ }^{3,4}$ Institute of Advanced Technology (ITMA), Universiti Putra Malaysia (UPM), Malaysia \\ ${ }^{5,6}$ Institute of Power Engineering (IPE), Universiti Tenaga Nasional (UNITEN), Malaysia
}

\begin{abstract}
Article Info
Article history:

Received Apr 26, 2019

Revised Jun 27, 2019

Accepted Jul 11, 2019

\section{Keywords:}

Charging platform

Charging systems

Electrical fundamentals

Landing

UAV

ABSTRACT

An efficient charging station is a necessity for Unmanned Aerial Vehicle (UAV) systems. However, if that implementation adds more complexity and onboard weight, then that exercise becomes a burden rather than a benefit since UAV's engineers aim to improve efficiency by reducing the energy consumed by the software and hardware of the complete aeronautical system. This article recommends a fully automatic contact charging station for UAVs, which can charge UAVs and thus resolve flight endurance restrictions of the UAV. The ground station consists of square copper plates that are positively and negatively polarized successively in a chessboard with particular sizes to guarantee electric contact at the landing. The design methodology used with the loading station takes into account the differences in UAV orientation once the platform has landed. In addition, this innovation uses independent charging after touchdown. Thus, this technology relaxes common flight times and help to enhance general mission times. This paper presents a unique charging platform in a "chessboard" configuration, which is devised as an interconnecting interface to facilitate the charging process and overcome inaccuracies with the landing. The solution devised in this research requires few components and presents two power source options (solar \& mains power). Additionally, this work presents, to the best of our knowledge, a uniquely innovative recharging landing platform, which incidentally requires no additional software or changes to the UAV's onboard software settings.
\end{abstract}

Copyright $@ 2020$ Institute of Advanced Engineering and Science. All rights reserved.

\section{Corresponding Author:}

Wan Zuha Wan Hasan,

Department of Electrical and Computer Engineering,

University Putra Malaysia,

Selangor, Serdang, 43400, Malaysia.

Email: wanzuha@upm.edu.my

\section{INTRODUCTION}

Lightweight electric Vertical Take-Off and Landing (VTOL) Unmanned Aerial Vehicles (UAVs) are becoming increasingly popular. These aerial vehicles have nearly limitless potential because of their compact size, which makes them affordable in cost, remote and safe solution [1]. A VTOL UAV's are ideal for various applications including surveillance, monitoring, and couriering [2]. UAVs can be loaded with varying amounts; typical payloads include high-resolution cameras, like infrared cameras, and other sensors. Notable applications for these UAVs include medical emergency scenarios since the UAV can be equipped with unique electronic identifier apparatus and provide an image of the type of medical situation [3]. Applied in forest fire scenarios, a UAV can be equipped with fire monitoring and fire suppression equipment $[4,5]$, which can aid in the efforts to detect early fires and help with the plan to suppress of forest fires at critical 
points to minimize the destruction, caused by the rapid convection propagation and long combustion cycle of the fire.

UAV's of rotor type lifts with high-speed propellers powered by brushless high-power DC engines. The hardware of the Multi-Copter Control System usually consists of a central processing unit and sensors to stabilize the UAV attitude such as gyroscopes and accelerometers And those circuits consume a certain amount of power. However, rotor engines represent the most considerable energy drain because multi-rotor UAVs must produce a lifting force to continuously move against gravity. This continuous flight drains of the power limits of the UAV multi-rotor operation [6,7]. Lithium-ion cells or batteries are often on-board power sources, but these high-power lithium batteries are commercially affordable to allow a flight time of approximately 20-40 minutes. [8,9]. It is therefore obvious that a multi-copter is required instantly after landing to extend the flight's time of operation This charging procedure traditionally needs direct human intervention and causes a delay before the vehicle can begin charging. To deal with this delay, an automatic charge process is required after the multi-copter landing on a charging station. the solution needs to start quick charging without human interference, In order to optimally decrease the charge time Other techniques are frequently used to extend the flight time [10] and to offer expanded tasks :

a) To supply the UAV with a higher capacity battery, but this certainly increases weight and thus reduces payload, or which will reduce the flight time of the UAV [11].

b) To build a docking technique to exchange batteries automatically when landing

c) $[12,13]$. This solution decreases UAV landing time but needs extra complicated mechanical components that increase costs and render the solution impractical [14].

d) To transmit the power to the UAV wirelessly, during flight mission [15]. One of the suggested solutions uses a laser beam with high energy. This technique is possible but expensive to use because powerful laser emission stations are necessary [16] but poses a serious health danger that could harm to human eyes [17], and hazard a breach of the Laser Safety Initiative of the Federal Aviation Administration (FAA) [18].

e) Another plausible method is to electromagnetically transmit power from a ground base to the UAV while in flight [19], For UAV apps, these systems may be omnidirectional and appealing. However, it is susceptible to environmental mitigation and energy efficiency problems [20].

f) Another method of wireless power transmission uses Wireless Power Transfer (WPT) technology. WPT is a popular technique employed in many low power charging applications for smartphones and electronic devices and works at a short-range on a changing station to charge by induction [21].

The application of WPT technology must consider the aerodynamic considerations of the onboard charging system and avoid increasing the overall weight of the aircraft. Also, the WPT module must not induce interference with other electronic systems of the UAV. Finally, the WPT technology must guarantee efficient power transfer even with a high a coil misalignment tolerance, which accounts for the inaccuracies of landing [22, 23]. A study WPT technology presented in [24] defines a system comprising of two circular planar spiral coils, the main disadvantages of that configuration is the significant increase in weight and physical dimensions of the components used. That system reduced the payload capacity of the drone and large components compromised the aerodynamic stability of the UAV. The maximum WPT charging efficiency is discussed in another article [25], that article reported an efficiency of $63.4 \%$, which varied with time. However, another article [26] reported experiments a better performance with a WPT system achieving a $75 \%$ charging efficiency.

Massachusetts institute of technology researchers [16] and ETH Zurich have developed an independent contact recharge station for UAVs. This charge solution involves contact with mechanical electrodes and the UAV must land on an accurate manner on the charging station, which ultimately increases the control complexity and therefore affects the costs of the entire system [27]. The system utilizes a Vicon Camera System which provides precise UAV and ground station position information, However, we should recognize that the Vicon Camera System cannot be used outside and that, instead, GPS sensors should be used despite inherent position mistakes [28]. These basic GPS sensor mistake[29] cause problems with platform docking and therefore increase the probability of the failure recharging.

A contract-based solution allows high power transfer efficiency, but one major drawback of contactbased charging is the requirement to precisely land on the charging station and the mechanical system in order to make contact with the electrodes to enable conduction, which eventually increases the control and complexity and cost [30, 31]. Furthermore, accurate UAV landing is often challenging even with a sophisticated targeting system, as that system must attempt to accurately compensate for the weather conditions experienced in outdoor environments[27, 29].

This paper offers an alternative solution for an automatic contact drone charging station using an innovative onboard charges circuit. This system enhances the autonomy of UAVs by avoiding human intervention to charge when landing. The charging process occurs when the Quadcopter air vehicle is landed 
on the ground platform and the contact terminals that already installed on each skid of the UAV are touched the ground platform, which is comprised of square shapes copper plates with specific dimensions and alternatively polarized The drone doesn't have to unplug from the landing pad and can rapidly take off unhindered. The drone can land in any direction with its skids in touch with the platform. Charging begins automatically without human interference.

\section{RESEARCH METHOD}

This work offers a solution to UAV's recharge system uncertainty through direct interaction with the ground charge platform configured as a chessboard with a successive copper platform with positive and negative polarities. The platform can be powered by two energy sources: indoors by DC adapters or outdoors by a solar panel. The primary parts of the system are shown in Figure 1.

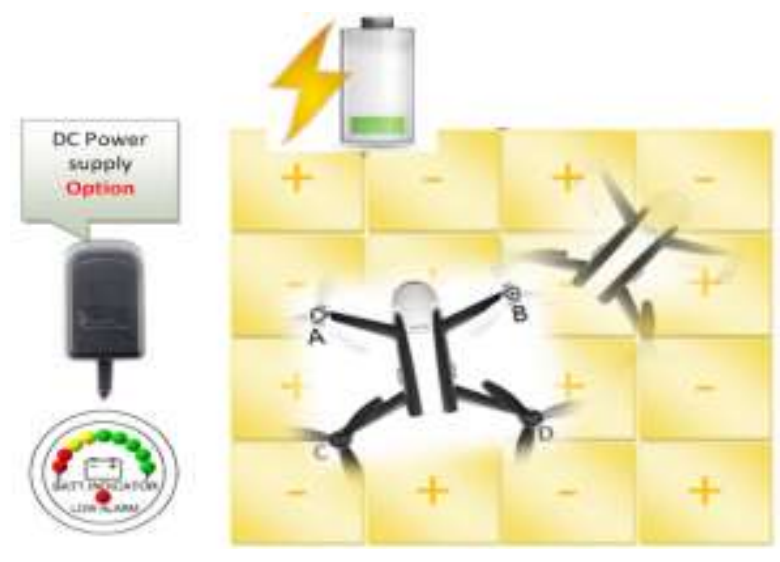

Figure 1. Main system components

\subsection{Pad Area Calculation}

A repeatability test of the UAV landing has been carried out in the course of 20 attempts to specify the region of the charging pad. the landing location is marked after each flight to track the UAV coverage region each time under the same flight circumstances. The aim of this experiment is to assess the peak landing error of the XY to calculate the size of the recharging pad at the ground station. The findings of these efforts are illustrated in Figure 2.

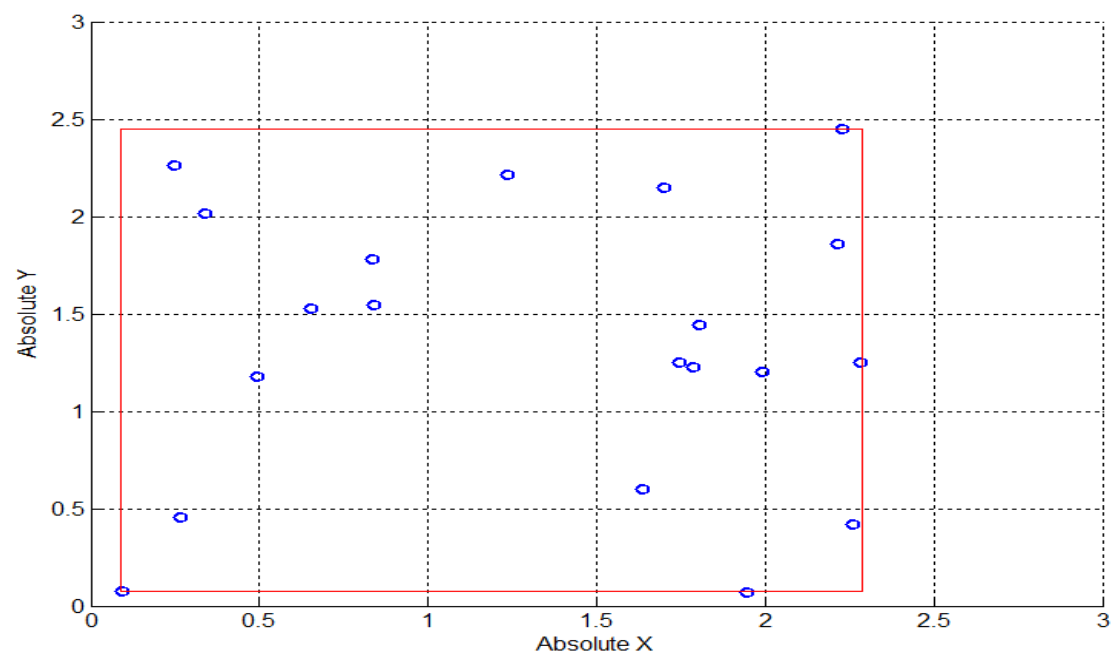

Figure 2. Charging pad area calculation process 


\subsection{On-Board Circuit Diagram}

A four contact pins are connected to the polarity modulator circuit, one for each leg of the drone. Six diode rectifiers are employed, all positive connected at one terminal and same for the negative, while the other two input terminals are linked with the four drone pins and have all probability of polarity adjustment. The two output terminals are connected to the main terminals of the onboard battery of the drone. The configuration diagram of the proposed system is shown in Figure 3.

Figure 3 shows the diode bridge setup, The configuration design of this component operates to supply a single polarity charging signal to the drone battery regardless of the drone angle of landing, the number of contact pins that should be attached to ground to achieve the charging process is restricted between (2-4), However, if each pin has a positive or negative polarity. The voltage at the drone battery terminals was adapted to a rate equal to its complete charge voltage considering the drop voltage of the forward diodes. Figure 4 shows the experimental configuration of the proposed framework. This system setup is designed to overcome misalignment circumstances due to landing defect. Drone landing accuracy is difficult to achieve because of the variability of the environment and the flight compensation required depends upon the accuracy of the landing assistance system. The on-board electronic settings are optimized to decrease the component weight, which reduces complexity and costs. In order to decrease the weight of the UAV, battery charging is regulated at the ground station.

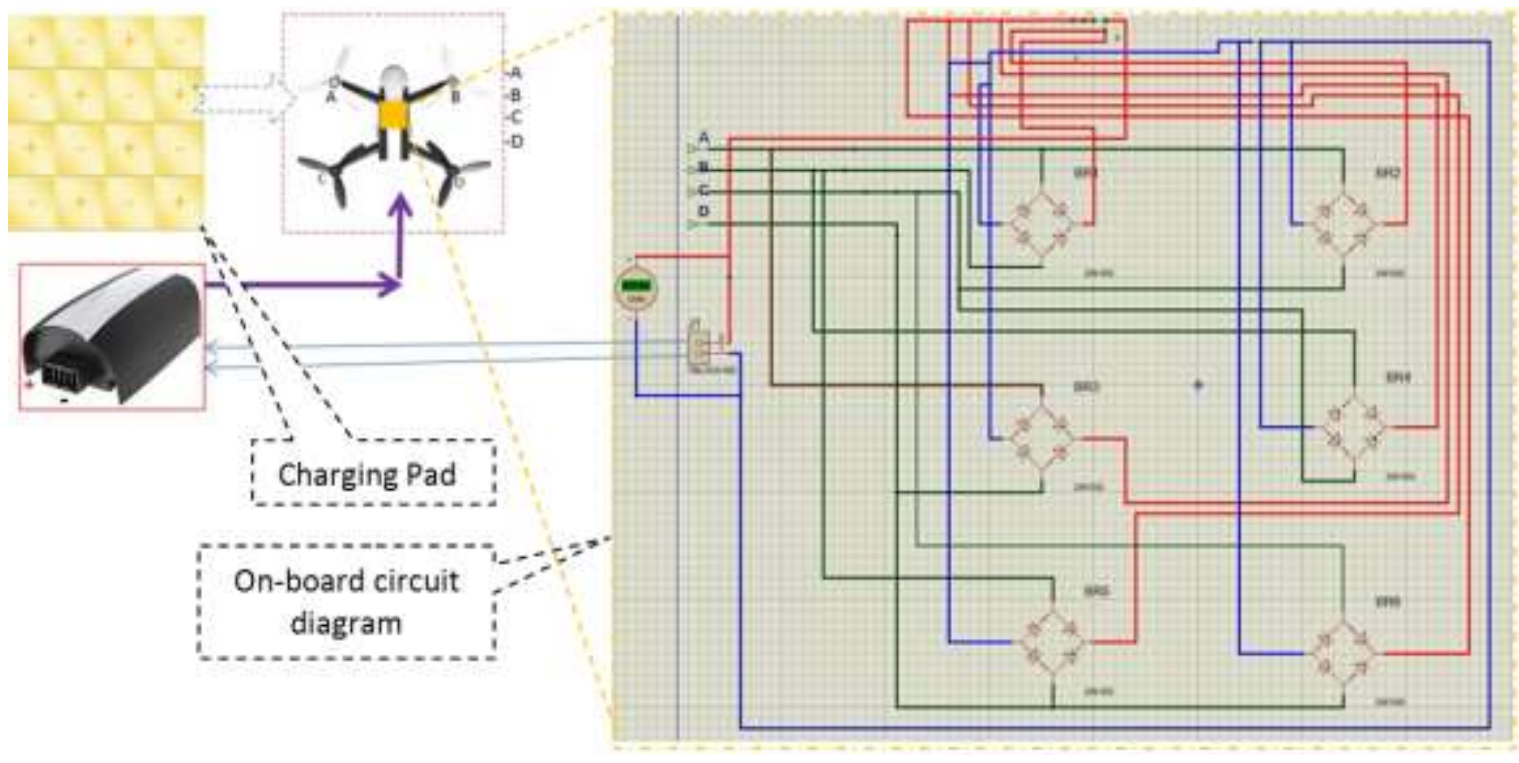

Figure 3. A configuration diagram of the proposed system.

\section{RESULTS AND ANALYSIS}

Due to the landing tolerance, this setup avoids any misalignment circumstances. This aspect is critical in outdoor drone apps as landing accuracy is difficult to accomplish owing to variability in environmental circumstances and intrinsic inaccuracies of landing support systems. The electronic circuits on board are optimized to decrease weight, circuit complexity, and cost. The battery charge regulation is carried out on the side of the supply (ground station) to decrease payload and size of the onboard equipment. The probabilities of the number of landings (Pc) can be displayed: $\mathrm{Pc}=4 !=24$. Where the disconnection pin occurrences are exclusive, that represent the landing on the chess-pad with less than 4 contacts.

\subsection{Charging Analysis}

The charge current and the voltage during the charging process is shown in Figure 5 and. Figure 6 respectively. A full charging last approximately $180 \mathrm{~min}$. naturally, the current during charging performance of the power supply starts for a drop with time as an indication of the successful charging process. To supply a regulated $12.5 \mathrm{~V}$ output the input voltage is set to $13.75 \mathrm{~V}$ this balances the full charge voltage of the onboard battery, However, the battery voltage shows rising during time as an indication of successful storage of power. 


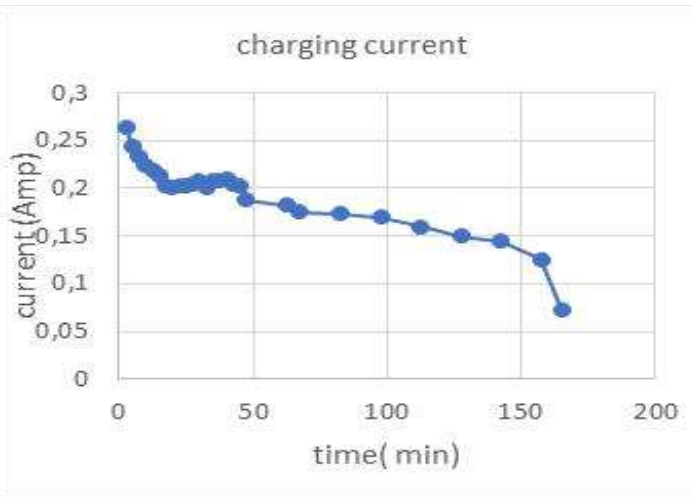

Figure 5. Charging current versus time

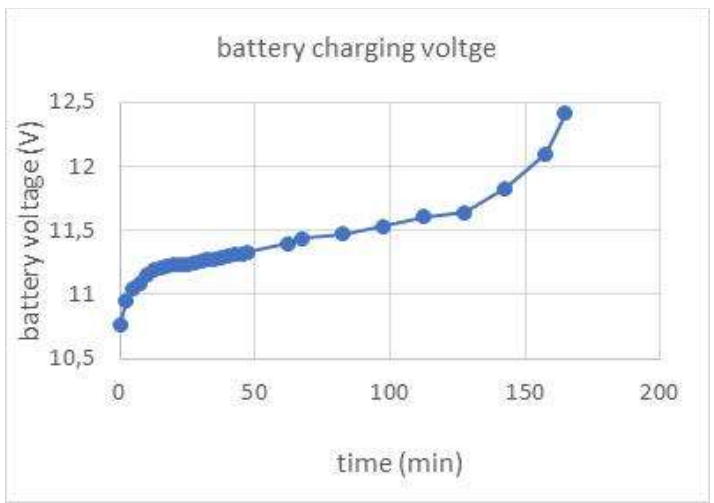

Figure 6. Onboard battery voltage during the charging process

\subsection{Charging Results Based on Power Supply}

To verify charging reliability and performance, we have investigated two types of energy providers. First, we have connected the charging, platform with a DC power supply to animate the standard commercial power supply. Second, we have connected the charging platform to the solar panel with dimensions of $1 \mathrm{~m} 2$ and a maximum power of $\mathrm{P}=100 \mathrm{~W}$. As shown in Figure 7and Figure 8, respectively.

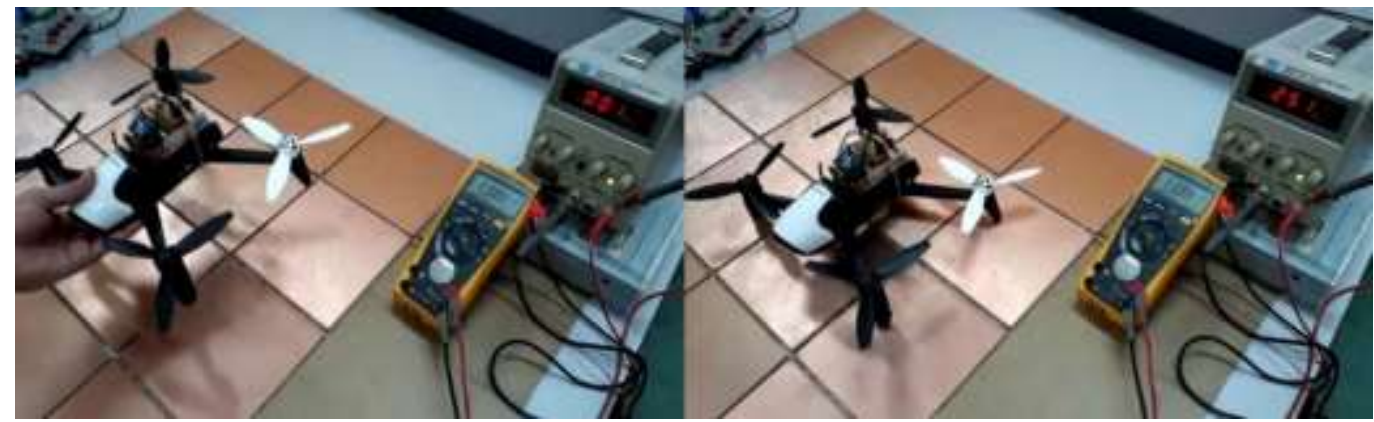

Figure 7. The test to ensure the reliability of the direct contacts method

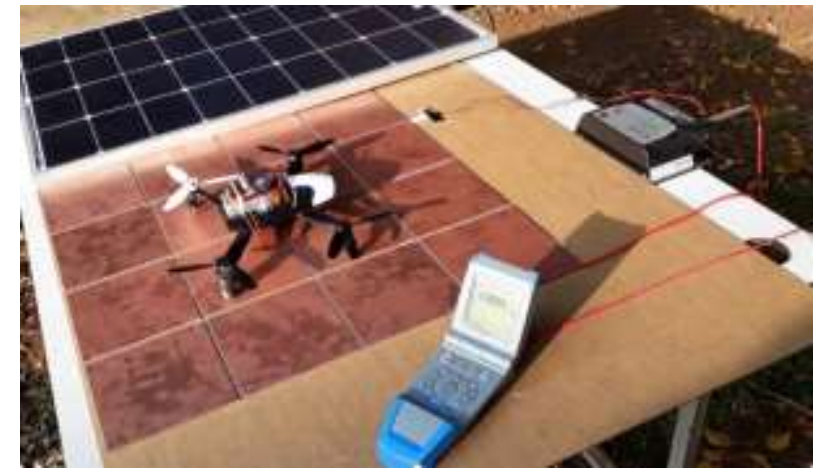

Figure 8. charging process powered by solar PV module

The test to ensure the reliability of the direct contacts method for charging the onboard drone battery. The outcome of the attempts positively contributed with the presented method, where a current flow from the power supply to the battery through the chessboard and the onboard contacts once the drone touches the charging platform. 


\section{CONCLUSION}

This study provides an optimum contact tile configuration for a UAV ground charging platform. All the UAV on-board components design to have minimum effect on flight aerodynamics and the circuit design is very compact in order to reduce the aircraft's weight. This research innovates six series of diode bridges linked to the drone skids and four spring contact pins. These spring contacts interface with the platform layout to start battery recharging effectively and autonomously upon landing This design of the landing pad, however, has constraints and is susceptible to issues caused by precipitation. Future work could be carried out to solve these problems. The layout of the landing pad could compensate for moisture problems to resist the load modifications between neighboring pads. In turn, present flow in the ground-based platform can be limited and protected against short circuits. Also, A heating component could also be added to evaporate humidity following the reduction of precipitation. Additional improvements are feasible for the autonomous outdoor and indoor energy sources for the ground stations. The development of this platform offers an effective and scalable UAV charging scheme.

\section{ACKNOWLEDGEMENT}

The authors acknowledge university Putra Malaysia (UPM) for supporting this research work under supervision and fund by FRGS Grant (5540076) and Kementerian Pelajaran Malaysia (KPM) and Institute of Advanced Technology (ITMA) in which this work was possible.

\section{REFERENCES}

[1] M. Hassanalian and A. Abdelkefi, "Classifications, applications, and design challenges of drones: A review," Prog. Aerosp. Sci., vol. 91, pp. 99-131, April, 2017.

[2] H. Kim, L. Mokdad, and J. Ben-Othman, "Designing UAV Surveillance Frameworks for Smart City and Extensive Ocean with Differential Perspectives," IEEE Commun. Mag., vol. 56, no. 4, pp. 98-104, 2018.

[3] M. Support and I. By, “( 12 ) United States Patent ( 45 ) Date of Patent :," vol. 1, no. 12, 2016.

[4] C. Yuan, Y. Zhang, and Z. Liu, "A survey on technologies for automatic forest fire monitoring, detection, and fighting using unmanned aerial vehicles and remote sensing techniques," Can. J. For. Res., vol. 45, no. 7, pp. 783-792, 2015.

[5] K. P. Valavanis and G. J. Vachtsevanos, "Handbook of unmanned aerial vehicles," Handb. Unmanned Aer. Veh., pp. 1-3022, 2015.

[6] C. H. Choi, H. J. Jang, S. G. Lim, H. C. Lim, S. H. Cho, and I. Gaponov, "Automatic wireless drone charging station creating essential environment for continuous drone operation," 2016 Int. Conf. Control. Autom. Inf. Sci. ICCAIS 2016, pp. 132-136, 2017.

[7] T. Dietrich, S. Krug, and A. Zimmermann, "An empirical study on generic multicopter energy consumption profiles," 11th Annu. IEEE Int. Syst. Conf. SysCon 2017 - Proc., 2017.

[8] P. Sarunic and R. Evans, "Hierarchical model predictive control of UAVs performing multitarget-multisensor tracking,” IEEE Trans. Aerosp. Electron. Syst., vol. 50, no. 3, pp. 2253-2268, 2014.

[9] B. Lee, S. Kwon, P. Park, and K. Kim, "Active power management system for an unmanned aerial vehicle powered by solar cells, a fuel cell, and batteries," IEEE Trans. Aerosp. Electron. Syst., vol. 50, no. 4, pp. 3167-3177, 2014.

[10] D. R. Dale, "Automated Ground Maintenance and Health Management for Autonomous Unmanned Aerial Vehicles," S.B. Phys., no. 2006, 2006.

[11] K. Neitzke, "Rotary Wing Micro Air Vehicle Endurance."

[12] D. Lee, J. Zhou, and W. T. Lin, “Autonomous battery swapping system for quadcopter,” 2015 Int. Conf. Unmanned Aircr. Syst. ICUAS 2015, pp. 118-124, 2015.

[13] K. A. O. Suzuki, P. Kemper Filho, and J. R. Morrison, "Automatic battery replacement system for UAVs: Analysis and design,” J. Intell. Robot. Syst. Theory Appl., vol. 65, no. 1-4, pp. 563-586, 2012.

[14] S. Jung, T. Lee, T. Mina, and K. B. Ariyur, "Inductive or Magnetic Recharging for Small UAVs," no. April 2016, 2012.

[15] T. Nugent and J. Kare, "Laser power for UAVs," Laser Motiv. White Pap., pp. 1-9, 2010

[16] N. K. Ure, G. Chowdhary, T. Toksoz, J. P. How, M. A. Vavrina, and J. Vian, "An automated battery management system to enable persistent missions with multiple aerial vehicles," IEEE/ASME Trans. Mechatronics, vol. 20, no. 1 , pp. 275-286, 2015.

[17] T. J. Nugent, Jr. and J. T. Kare, "Laser power beaming for defense and security applications," p. 804514, 2011.

[18] Omar I. Dallal Bashi, W. Z. Wan Hasan, N. Azis, S. Shafie and Hiroaki Wagatsuma, "Autonomous Quadcopter Altitude for Measuring Risky Gases in Hazard Area" Journal of Telecommunication, Electronic and Computer Engineering, pp. 31-34, 2018

[19] T. Campi, S. Cruciani, and M. Feliziani, "Wireless Power Transfer Technology Applied to an Autonomous Electric UAV with a Small Secondary Coil," Energies, vol. 11, no. 2, pp. 352, 2018.

[20] C. Song et al., "EMI Reduction Methods in Wireless Power Transfer System for Drone Electrical Charger Using Tightly Coupled Three-Phase Resonant Magnetic Field," IEEE Trans. Ind. Electron., vol. 65, no. 9, pp. 6839-6849, 2018 . 
[21] A. Kurs, A. Karalis, R. Moffatt, J. D. Joannopoulos, P. Fisher, and M. Soljacic, "Supporting: Wireless power transfer via strongly coupled magnetic resonances.," Science, vol. 317, no. 5834, pp. 83-86, 2007.

[22] G. a. Covic and J. T. Boys, "Inductive Power Transfer," Proc. IEEE, vol. 101, no. 6, pp. 1276-1289, 2013.

[23] N. Shinohara, "Power Without Wires," IEEE Microw. Mag., vol. 12, no. 7, pp. S64-S73, 2011.

[24] T. Campi, F. Dionisi, S. Cruciani, V. De Santis, M. Feliziani, and F. Maradei, "Magnetic field levels in drones equipped with Wireless Power Transfer technology," 2016 Asia-Pacific Int. Symp. Electromagn. Compat. APEMC 2016, pp. 544-547, 2016.

[25]. Muhammad Ramez, Mohd Amrallah Mohd Mustafa, Wan Zuha Wan Hasan, Suhaidi Shafie; "Efficient Charging Pad for Unmanned Aerial Vehicle Based on Direct Contact", IEEE 5th International Conference on Smart Instrumentation, Measurement and Applications (ICSIMA), 2018.

[25] A. Bin Junaid, Y. Lee, and Y. Kim, "Design and implementation of autonomous wireless charging station for rotary-wing UAVs,” Aerosp. Sci. Technol., vol. 54, pp. 253-266, 2016.

[26] A. Bin Junaid, A. Konoiko, Y. Zweiri, M. N. Sahinkaya, and L. Seneviratne, "Autonomous wireless self-charging for multi-rotor unmanned aerial vehicles," Energies, vol. 10, no. 6, pp. 1-14, 2017.

[27] J. Leonard, A. Savvaris, and A. Tsourdos, "Energy Management in Swarm of Unmanned Aerial Vehicles," J. Intell. Robot. Syst., vol. 74, no. 1-2, pp. 233-250, 2014.

[28] R. M. Faheem, S. Aziz, A. Khalid, and M. Bashir, "UAV Emergency Landing Site Selection System using Machine Vision," vol. 1, no. 1, pp. 13-20, 2016.

[29] A. H. Sabry, W. Z. Wan Hasan, M. Zainal, M. Amran, and S. B. Shafie, "Alternative Solar-Battery Charge Controller to Improve System Efficiency,” Appl. Mech. Mater., vol. 785, no. February 2016, pp. 156-161, 2015.

[30] A. H. Sabry, W. Z. W. Hasan, M. Z. A. Ab Kadir, M. A. M. Radzi, and S. Shafie, "DC-based smart PV-powered home energy management system based on voltage matching and RF module," PLoS One, vol. 12, no. 9, 2017.

[31] T. S. Gunawan, I. R. H. Yaldi, M. Kartiwi, H. Mansor, "Performance Evaluation of Smart Home System using Internet of Things," International Journal of Electrical and Computer Engineering, vol. 8, pp. 400-411, 2018. 